Proceedings of the XXI International Meeting on Radio and Microwave Spectroscopy RAMIS 2005, Poznań-Będlewo, Poland, April 24-28, 2005

\title{
Mobile NMR for Analysis of Polyethylene Pipes
}

\author{
B. Blümich ${ }^{a}$, F. Casanova ${ }^{a}$, A. Buda $^{a}, \mathrm{~K} \cdot \mathrm{Kremer}^{a}$ \\ AND T. WEGENER ${ }^{b}$ \\ ${ }^{a}$ Institute of Technical and Macromolecular Chemistry \\ RWTH Aachen University \\ Worringerweg 1, 52056 Aachen, Germany \\ ${ }^{b}$ Institut für Rohrleitungsbau, FH Oldenburg \\ Industriestr. 11, 26121 Oldenburg, Germany
}

\begin{abstract}
NMR relaxometry is a suitable tool to determine the morphology of semi-crystalline polymers by its ability to discriminate between rigid, mostly crystalline and soft, usually amorphous material. The NMR-MOUSE ${ }^{\circledR}$ (nuclear magnetic resonance mobile universal surface explorer) was explored in this work to supply morphological data of poly(ethylene) pipes nondestructively. PE-100 pipes were investigated in the new state, after squeezing them flat, and after annealing well below the glass temperature. Furthermore, the change in morphology induced by a pressure load from the inside and a point load from the outside was investigated as a function of depth, and the morphology change across a welding line was imaged. A shear-band was detected by destructive high-field NMR imaging in an area of severe deformation of a pipe, where an anomalous depths profile was observed by the NMR-MOUSE. These results demonstrate that the NMR-MOUSE is a suitable tool for non-destructive state assessment of polymer pipes on the basis of laboratory reference data.
\end{abstract}

PACS numbers: 81.05.Lg, 82.35.Lr, 76.60.--k

\section{Introduction}

Polymer pipes are widely used for gas and water transport. A material of high commercial interest is high density polyethylene (PE), and the testing procedures for the prediction of their lifetime are spelled out in norms and regulations [1]. A fundamental issue of discussion is the laboratory testing conditions for accelerated aging which come closest to the deterioration of the pipes in actual use. 
The macroscopic properties of semi-crystalline polymers are determined by the morphology which changes at elevated temperature due to annealing and at elevated stress due to creep [2]. For lifetime predictions, polymer pipes are tested under pressure at an elevated temperature of $80^{\circ} \mathrm{C}$, and the results are then extrapolated to room temperature based on an Arrhenius relationship [3]. Wetting agents are used to approximate the conditions encountered in real use [4].

However, the lifetime prediction of PE pipes buried in the ground is still under discussion $[5,6]$, and to answer the question how far the conditions of accelerated laboratory aging defined in different norms and regulations are a sufficiently good approximation for the material deterioration under conditions of actual use, further investigations with methods sensitive to the different aspects of the polymer morphology are required. NMR relaxometry in combination with differential scanning calorimetry (DSC) has been found recently to be a powerful combination of methods for studying the effects of pressure and temperature on the morphology and the lifetime of poly(propylene) pipes [7]. While the investigations reported by Litvinov and Soliman were conducted in the laboratory on samples taken from pipes, the NMR-MOUSE ${ }^{\circledR}[8]$ is capable of providing similar data, albeit with lower signal-to-noise ratio, in a non-destructive fashion, opening up the perspective to quantify the state of PE pipes and to predict their lifetime on the basis of reference data. To substantiate this claim we have conducted first experiments with the NMR-MOUSE on PE pipes [9].

$\mathrm{PE}$ is a partially crystalline material consisting of crystalline lamellae with folded polymer chains and amorphous regions, where the polymer chains are expected to be randomly coiled. Yet, depending on the processing conditions, the orientation of the chain segments in the amorphous domains is not necessarily random. Due to shrinkage upon cooling and mechanical load, local order can be introduced. Furthermore, the polymer chains in the crystalline lamellae are by no means absolutely rigid, but can diffuse through the crystalline domains giving rise to creep and stress relaxation [10]. In addition to temperature, these molecular dynamics are further modified by the contact and ingress of solvent molecules. It is therefore clear that the material properties depend on the morphology, in particular the crystallinity and the order in the amorphous domains, which in turn depend on the mechanical and thermal history of the object and its contact with solvents and gases. Further material changes can be induced chemically, for example, by reaction of chain defects and residual catalyst with permeating oxygen and other chemicals.

\section{Experimental procedure}

Commercial PE-100 water pipes were investigated at room temperature with the NMR-MOUSE, a mobile unilateral NMR sensor which collects the NMR signal in the stray field from a region outside the device (Fig. 1) [8, 11]. The NMR-MOUSE is operated by a portable spectrometer which provides the radio- 


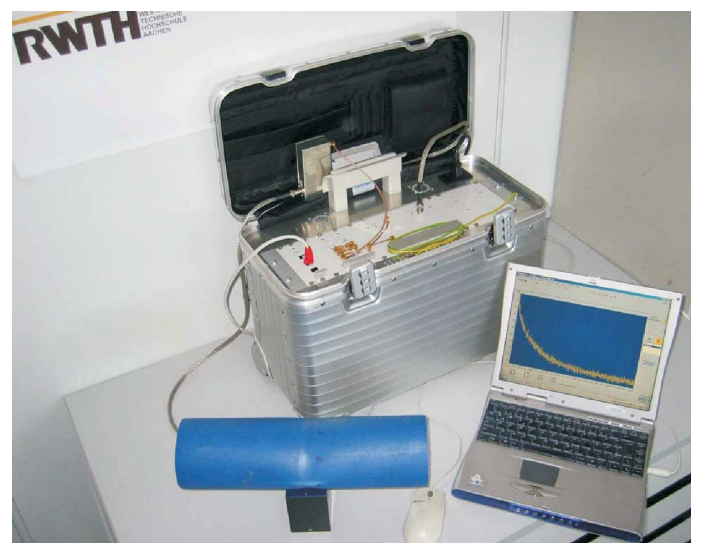

Fig. 1. Mobile NMR for nondestructive testing of PE pipes. The NMR-MOUSE is visible underneath the pipe. The NMR spectrometer for operation of the sensor is in the pilots case next to the pipe. The spectrometer is operated by a notebook computer.

-frequency (rf) excitation pulses and collects the data. As the measurement is conducted in inhomogeneous polarization and rf fields, the envelope of a CarrPurcell-Meiboom-Gill (CPMG)-type multi-echo train is acquired [11]. In weakly inhomogeneous fields, the CPMG echo envelope corresponds to the envelope of the free induction decay in a perfectly homogeneous field, and for a mono-exponential decay, the decay time constant would be the transverse relaxation time $T_{2}$. In strongly inhomogeneous fields, the flip angle distribution gives rise to a superposition of the Hahn and stimulated echoes in the multi-echo train, and the signal decay is determined by effective transverse relaxation time $T_{2 \text { eff }}>T_{2}$ [12].

For PE, the echo envelope measured with the NMR-MOUSE can be well approximated by a bi-exponential function

$$
y=y_{0}+A_{\text {short }} \exp \left(-t / T_{2 \text { eff, short }}\right)+A_{\text {long }} \exp \left(-t / T_{2 \text { eff, long }}\right) .
$$

The experimental data (Fig. 2a) are evaluated in either of two ways (Fig. 2b). For data with a good signal-to-noise ratio, the relative amplitude ratio $A_{\text {short }} /\left(A_{\text {short }}+A_{\text {long }}\right)$, and the relaxation rates $1 / T_{2 \text { eff, short }}$ and $1 / T_{2 \text { eff, long are }}$ determined from a fit of Eq. (1) to the measured data and interpreted in terms of the crystallinity, a measure of the average size of the crystallites, and a measure of the order in the amorphous domains, respectively. Let us note that the NMR crystallinity is defined via the segmental mobility and may include signal from rigid amorphous domains, thus differing from the X-ray crystallinity. Alternatively, a relaxation weight can be calculated from suitably selected partial sums of echoes $y_{i}$ (Fig. 2b). For example, the weight function

$$
w(2, k, l, m)=\sum_{i=1}^{k} y_{i} /\left(\sum_{i=2}^{k} y_{i}+\sum_{i=l}^{m} y_{i}\right)
$$



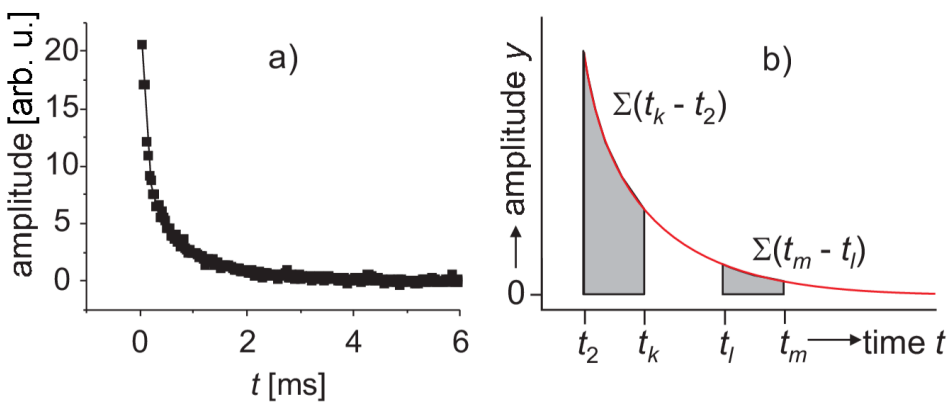

Fig. 2. (a) Experimental NMR signal from PE. Each point corresponds to the maximum of an NMR echo. (b) Principle of data evaluation for calculation of a relaxation weight. The sums defined in Eq. (2) are represented in terms of integrals as areas underneath the decay curve.

scales between 0 and 1 and is large for morphologies with a high content in rigid domains and is small for morphologies with a high content in mobile domains, as long as the partial sums are suitably placed at the beginning and in the middle of the decay. The relaxation weight is another measure of the crystallinity, which is easier to compute than a fit as no particular functional dependence of the signal decay needs to be assumed. But it depends on the summation limits and the echo time $t_{\mathrm{E}}$ used for measurement.

\section{Results}

To explore the use of the NMR-MOUSE for non-destructive testing of PE pipes, an extreme deformation, here a full compression, of a blue water pipe (PE 100, SDR 17) with an outer diameter of $110 \mathrm{~mm}$ and a wall thickness of $7 \mathrm{~mm}$ was investigated. Such deformations are permitted by the regulations to turn off the water flow for repair work. The NMR-MOUSE used operated at a frequency of $13.7 \mathrm{MHz}$ in a depth of $1 \mathrm{~mm}$. The pipe was measured at 8 points placed in equidistant steps on the circumference. Then the compression was executed for 3 days following the procedures spelled out in sheet GW 332 of DVGW with a commercial device (Fig. 3a) in such a way that points 1 and 5 marked the positions of extreme deformation. There, crazes were observed on the outside wall as a result of the deformation. Following the deformation, the pipe was returned into the round original state and measured again at the same points. Subsequently the pipe was annealed at $80^{\circ} \mathrm{C}$ for $24 \mathrm{~h}$ and after cooling measured again at room temperature.

Already the new pipe showed a scatter of about $12 \%$ in the fit parameters $1 / T_{2 \text { eff,long }}, 1 / T_{2 \text { eff,short }}$, and $A_{\text {short }} /\left(A_{\text {short }}+A_{\text {long }}\right)$ (Figs. $\left.3 \mathrm{~b}-\mathrm{d}\right)$. This scatter is reproducible and in accordance with the existence of local strain in the pipe. The effects remaining from the deformation shorten the values of $1 / T_{2 \text { eff, short }}$ (Fig. $3 \mathrm{~d}$ ). 

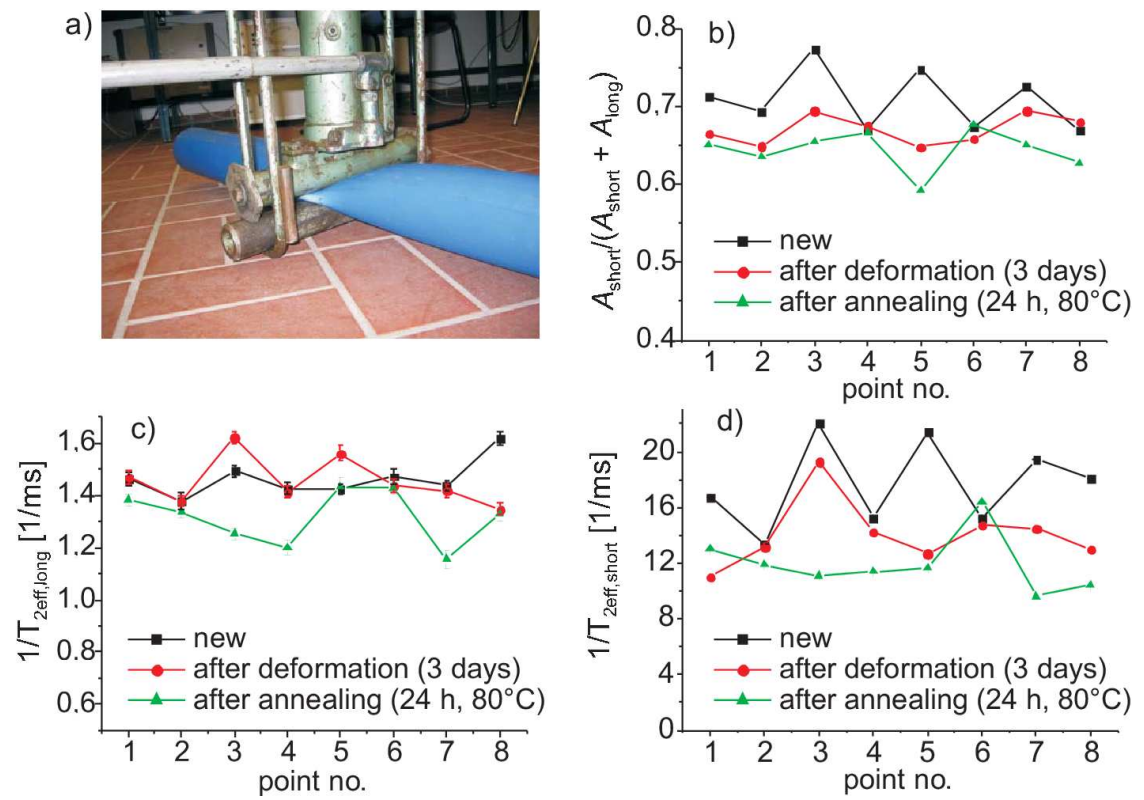

Fig. 3. Results of measurements on a water pipe with an outer diameter of $110 \mathrm{~mm}$ (deformation-annealing). (a) Deformation, (b) relative amplitude ratio, (c) $1 / T_{2 \text { eff, long, }}$, (d) $1 / T_{2 \text { eff } \text {,short }}$.

This is explained with a decrease in the mean size of the crystallites. A slow relax-

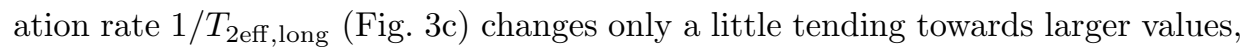
which is interpreted as a small increase in the molecular order in the amorphous domains. The NMR degree of crystallinity $A_{\text {short }} /\left(A_{\text {short }}+A_{\text {long }}\right)($ Fig. $3 \mathrm{~b})$ is reduced by the deformation, as the strain imposed on the chains in the amorphous domains by the deformation is relaxed by chains creeping out of the crystalline domains. Deformations for only $30 \mathrm{~min}$ did not lead to noticeable changes in the NMR parameters, indicating that this time is too short for appreciable stress relaxation at room temperature. Subsequently the pipe was annealed at $80^{\circ} \mathrm{C}$, i.e. about $40^{\circ} \mathrm{C}$ below the melting temperature of the ideal PE crystallite, to test how far the material changes induced by the deformation could be reversed. This temperature was chosen, as it is typically used in accelerated failure testing for lifetime predictions of $\mathrm{PE}$ pipes. Figures $3 \mathrm{~b}$ to d demonstrate that $1 / T_{2 \text { eff, long }}$ is reduced by the annealing, and the material clearly changes its properties by the

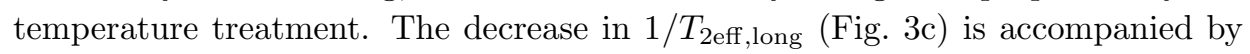
a decrease in the NMR crystallinity (Fig. 3b). This is explained with decreasing segmental order in the amorphous domains on the expense of losing small and defect-rich crystallites.

In a second series of tests (Fig. 4) with a similar pipe, the pipe was annealed first for $24 \mathrm{~h}$ at $80^{\circ} \mathrm{C}$ and deformed afterwards for 3 days. An increase in 
a)
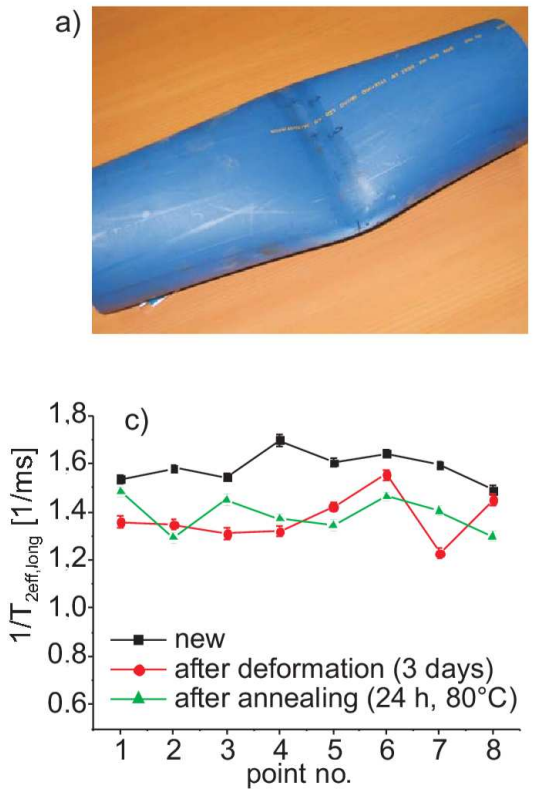
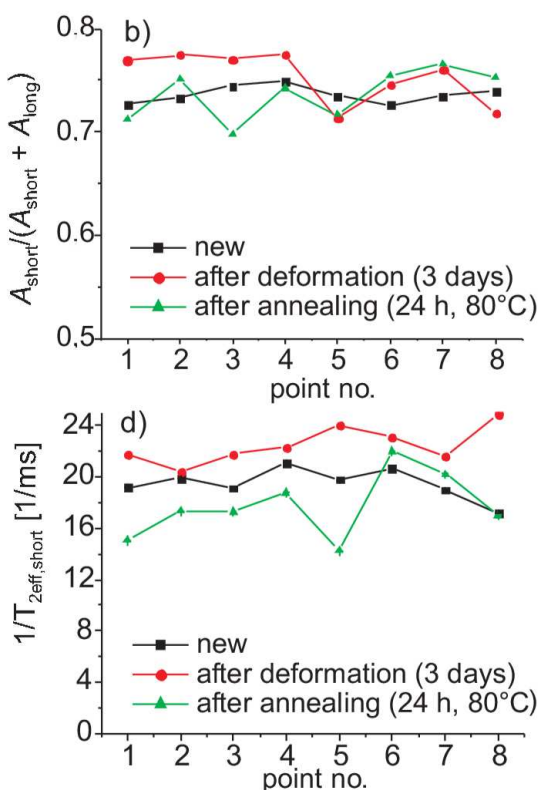

Fig. 4. Experimental results from a water pipe with an outer diameter of $110 \mathrm{~mm}$ (annealing-deformation). (a) Deformed pipe after annealing and deformation, (b) relative amplitude ratio, (c) $1 / T_{2 \text { eff,long }}$, (d) $1 / T_{2 \text { eff, short }}$.

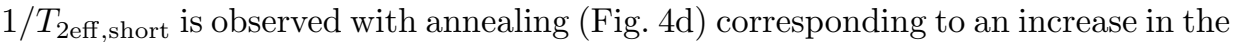

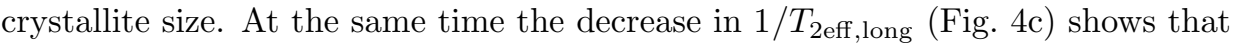
order in the amorphous domains is reduced as local stress is relaxed. The increase in the NMR crystallinity (Fig. 4b) upon annealing below the melting temperature of the ideal crystal is explained with a recrystallization of small crystallites and a growth of small crystallites on the expense of a loss of mobile amorphous material. The larger crystallinity after annealing explains the observation that the pipe could not completely be reshaped into its original circular form after deformation and appeared stiffer than before (Fig. 4a). After deformation, $1 / T_{2 \mathrm{eff} \text {,short }}$ is reduced in all measurement positions (Fig. 4d) with a particularly large effect in positions 1 and 5 where the deformation was strongest. No significant changes are observed in $1 / T_{2 \text { eff, long }}$ (Fig. 4c) indicating that the amorphous regions are not much affected in this case.

In another series of measurements two further, blue PE-100 water pipes of the same kind were investigated in an internal pressure test with an external point load. Before the test, pipe 1 was mapped from the inside with a specially designed NMR-MOUSE on a $1 \mathrm{~cm}^{2}$ grid. The NMR-MOUSE and the measurement results are depicted in Fig. 5. The parameter plotted is the weight function according to Eq. (2) in a measurement depth of $0-1 \mathrm{~mm}$ acquired with an echo time $t_{\mathrm{E}}=$ $25 \mu \mathrm{s}$. From an axial trace through the 2D map at $2 \mathrm{~cm}$ circumference it is clear 


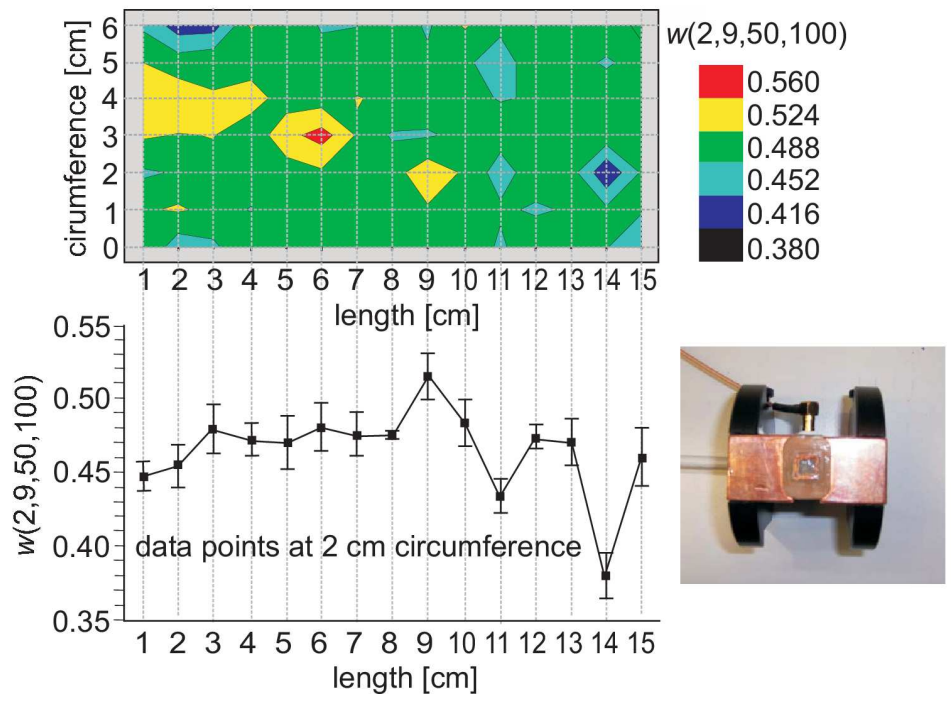

Fig. 5. Map of the relaxation weight for a section in the center of pipe 1 on a $1 \mathrm{~cm}^{2}$ grid (top). At some positions, the measured values clearly deviate from the mean. The axial trace at $2 \mathrm{~cm}$ circumference is plotted with error bars to compare the variation with the measurement error (bottom). The data were acquired with a special NMR-MOUSE constructed for measuring inside a pipe (bottom right).

that the values vary beyond the experimental error. At each position the weight function was measured three times, and the average and the standard deviation were computed. For reasons of simplicity, the mean value is denoted as $w$. At $9 \mathrm{~cm}, w$ assumes a much higher value in the selected trace and at $14 \mathrm{~cm}$ a much lower value than the mean value from the different positions of the pipe. Clearly, the pipe is inhomogeneous in agreement with the results depicted in Figs. 3 and 4.

After mapping pipe 1 at different positions, the pipe was tested according to Kiesselbach [13] with external point loads and internal water pressure (Fig. 6). The testing temperature was $+5^{\circ} \mathrm{C}$. At three positions external point loads were applied with pins having half spherical caps of $10 \mathrm{~mm}$ diameter deforming the unpressurized pipe by $0 \%, 1 \%$, and $6 \%$. Then the internal water pressure was
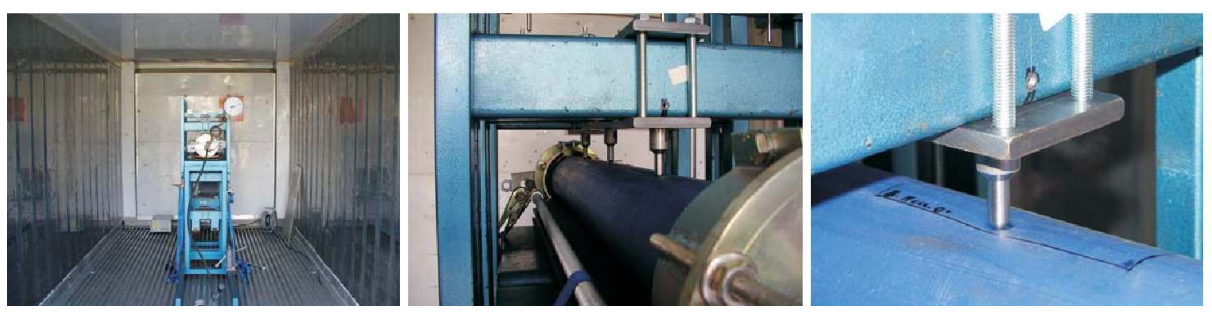

Fig. 6. Setup at the University of Oldenburg for conducting the pressure test with an external point load according to Kiesselbach. 

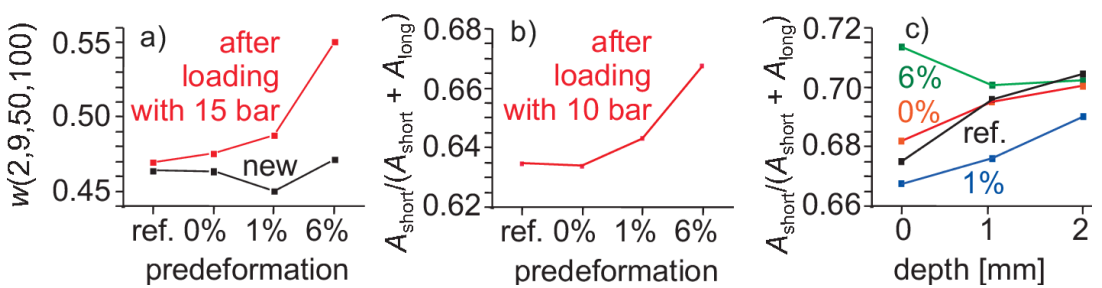

Fig. 7. Measured material changes on pipes subjected to the internal pressure test with an external point load. (a) Pipe $1, t_{\mathrm{E}}=25 \mu \mathrm{s}$. (b) Pipe $2, t_{\mathrm{E}}=45 \mu \mathrm{s}$. The deformation-dependent data were measured from the inside with the NMR-MOUSE depicted in Fig. 5. (c) Material changes in pipe 2 at different depths. The measurements were conducted with a different NMR-MOUSE from the outside and a different echo time, so that the values on the abscissae of parts (b) and (c) are not comparable in absolute numbers.

turned on for $24 \mathrm{~h}$ to 15 bar, which is by a factor of 1.5 higher than the maximum value allowed for the operation of drinking water pipes. Following the pressure test, the same positions were measured again with the NMR-MOUSE from the inside. The values obtained before and after the pressure test are compared in Fig. 7a. The reference measurements on a point outside the deformation region produce the same values before and after the test. Similarly, the values for $0 \%$ deformation are close but not identical as the pipe expanded upon pressurizing. However, already for the $1 \%$ deformation, the weight function $w$ changes significantly, and a $17 \%$ change is observed for $6 \%$ deformation. This is in agreement with finite element simulations by Kiesselbach [13], who predicted a beginning damage in the inner pipe wall for this situation.

Pipe 2 was also investigated in the pressure test with a point load and three deformations but with a lower inner pressure of 6 bar corresponding to the 1.5 fold of the maximum value allowed for gas pipes. Furthermore, instead of the relaxation weight $w$ (Eq. (2)) the amplitude ratio (Eq. (1)) was evaluated (Fig. 7b). The same trend of the data is observed as in Fig. 7a, but the change in the amplitude ratio is only about $6 \%$ at $6 \%$ deformation in agreement with a lower pressure load.

The pressure points of pipe 1 were further investigated by depth-dependent measurements with a conventional NMR-MOUSE from the outside. For the reference point, for $0 \%$, and for $1 \%$ deformation, the amplitude ratios increase with depth changing from 0 to $2 \mathrm{~mm}$. This corresponds to NMR crystallinity increasing from the outside to the inside. The values for the reference point and for $0 \%$ deformation are about the same. Those for $1 \%$ deformation are somewhat lower, which can be explained by material inhomogeneities. However, the values for $6 \%$ deformation are very different. Here the amplitude ratio decreases with depth, indicating a significant and unexpected change in the material properties.

An increase in the amplitude ratio or in $w$ correlates with reduced segmental mobility of the macromolecular chains (cf. Eqs. (1) and (2)), in agreement with the 
expected crystallinity gradient induced by cooling of the pipe during manufacturing and with the NMR-crystallinity gradient observed for increasing deformations (Figs. 7a, b). The data in Fig. 7a therefore confirm that the areas of high $w$ in the map of pipe 1 (Fig. 5) can be interpreted as regions of increased local strain.

The section of the pipe 1 exposed to $6 \%$ deformation, which showed the unexpected depth profile (cf. Fig. 7c) was cut out from the pipe and investigated at $7 \mathrm{~T}$ by 3D single-point imaging (Fig. 8) [11]. The field of view was $20 \mathrm{~mm} \times$ $20 \mathrm{~mm} \times 20 \mathrm{~mm}$, with a resolution of $312 \mu \mathrm{m}$ in all space directions. Images $8 \mathrm{c}-\mathrm{i}$ are $2 \mathrm{D}$ slices through the $3 \mathrm{D}$ image of the sample (Fig. 8a) parallel to the pipe wall. Light colors correspond to regions with higher segment mobility than dark colors. The darker the color, the more restricted is the segmental mobility.
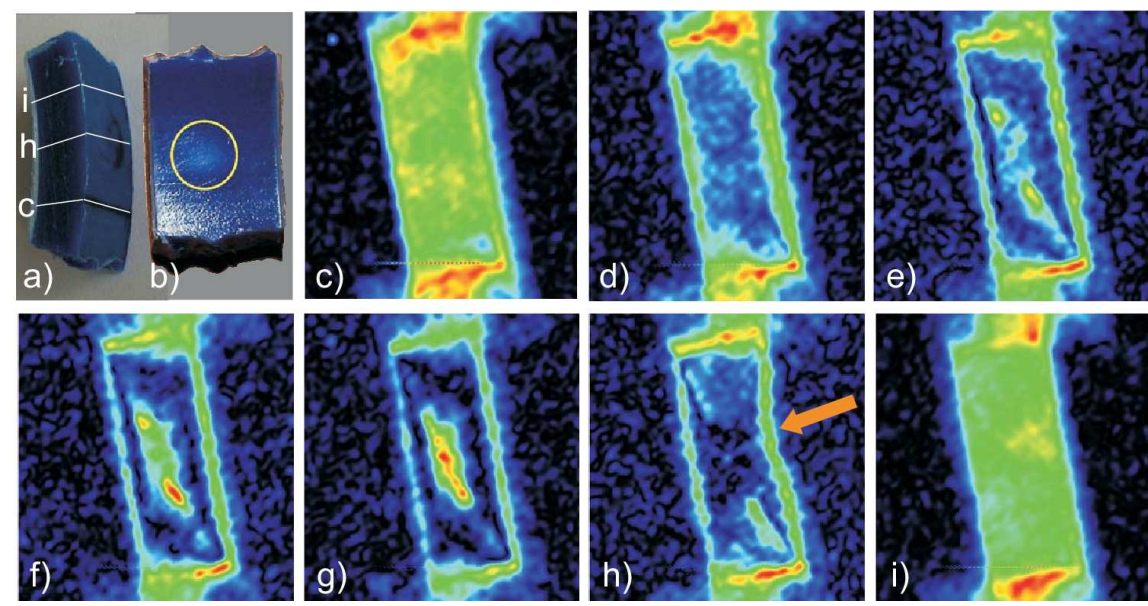

Fig. 8. NMR images of the section of PE pipe 1 with $6 \%$ deformation. (a) Photo of the section cut out from the pipe with marks indicating the position of the slices. (b) View from the inside of the pipe showing stress whitening of the material. (c)-(i) $2 \mathrm{D}$ slices through the 3D NMR image. The arrow in (h) indicates the center of the external point load.

The images demonstrate that the segmental mobility is lower inside the pipe wall than at both surfaces (cf. Fig. 8d). This is in accordance with an increase in the weight parameter $w$ with increasing depth (Fig. 7c). Most remarkable, however, is the formation of a complex shear band in the center of the deformation from the point load (Figs. 8e-h) with hard (dark) and soft (light) regions. At the inside of the pipe stress whitening is observed as a consequence of the deformation (Fig. 8b). The occurrence of shear banding and stress whitening is not mutually exclusive [11]. Which effect dominates depends on the stress conditions and the temperature.

To complement the investigations of PE-100 pipes, the question was addressed, if welding lines can be detected by the NMR-MOUSE. To this end, an 


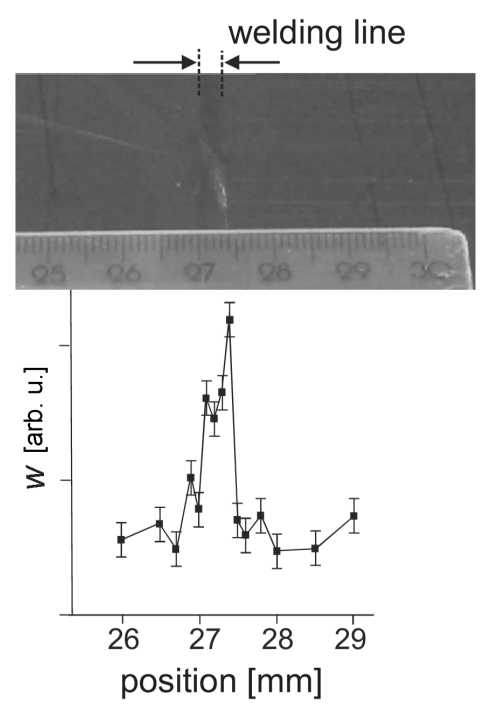

Fig. 9. 1D image across a welding line of two PE plates scanned in a pointwise fashion by lateral displacement of an NMR-MOUSE.

NMR-MOUSE was constructed with a narrow coil to reduce the width of the sensitive volume, and a lateral profile across the welding line was measured at a depth of $1 \mathrm{~mm}$. Within the area of the welding line, the relaxation weight $w$ is higher than outside, indicating higher NMR crystallinity. The spatial resolution is remarkably good, because the width of the imaged profile is only slightly larger than the width of the welding line (Fig. 9). Further investigations are needed to correlate the NMR signal with the quality of the welding line and to develop the NMR-MOUSE into a tool for safety inspection of welding lines.

\section{Summary}

The NMR-MOUSE has been shown to be suitable to characterize morphological changes in semi-crystalline polymers non-destructively. PE-100 pipes were investigated with the NMR-MOUSE with regard to material inhomogeneities and material changes before and after deformation and annealing at $80^{\circ} \mathrm{C}$ as well as before and after pressure tests with a point load. The results demonstrate that the associated changes in the semi-crystalline morphology can be followed by the NMR-MOUSE and consistently be explained. High-field NMR imaging revealed the formation of a shear band in a zone of high deformation by a point load. The investigations conducted so far serve to illustrate the potential use of mobile NMR for non-destructive testing of PE pipes. In particular, they demonstrate that this methodology is sufficiently sensitive to material changes and can be used for non-destructive state assessment of PE pipes in the field. This substantiates the perspective to conduct lifetime predictions on the basis of non-destructive field 
tests and laboratory reference data. Moreover, the method can potentially be useful for safety inspection of welding lines in polymer products.

\section{Acknowledgments}

The authors thank M. Adams, K. Münnemann, and A. Amar at ITMC of RWTH Aachen for their help with the NMR-MOUSE measurements and the single-point imaging measurements as well as T. Wegner, M. Böge, and M. Heyer at IRO Oldenburg for conducting the pressure tests with a point load. The stimulating initiatives and interesting comments of $\mathrm{H}$. Wagener to this work are gratefully acknowledged.

\section{References}

[1] See for example DVGW-Arbeitsblatt $G 401$ and DVGW-Hinweis $W 401$.

[2] D.W. van Krevelen, Properties of Polymers, Elsevier, Amsterdam 1990.

[3] Rohrsysteme aus PE 100, Ed. H. Brömstrup, Vulkan-Verlag, Essen 2004.

[4] J. Hessel, 3R International 40, 178, 360 (2001).

[5] H. Krietenbrink, R. Kloth, 3R International 43, 576 (2004).

[6] R. Grosse-Boes, R. Kloth, 3R International 43, 233 (2004).

[7] V.M. Litvinov, M. Soliman, Polymer 46, 3077 (2005).

[8] G. Eidmann, R. Savelsberg, P. Blümler, B. Blümich, J. Magn. Reson. A 122, 104 (1996).

[9] B. Blümich, F. Casanova, A. Buda, K. Kremer, T. Wegener, 3R International, in press.

[10] G. Strobl, The Physics of Polymers, Springer, Berlin 1996.

[11] B. Blümich, NMR Imaging of Materials, Clarendon Press, Oxford 2000.

[12] F. Balibanu, K. Hailu, R. Eymael, D.E. Demco, B. Blümich, J. Magn. Reson. 145, 246 (2000).

[13] G. Kiesselbach, gwf-Wasser/Abwasser 145, 45 (2004). 